\title{
Stress relaxation of wood partially non-crystallized using aqueous $\mathrm{NaOH}$ solutions
}

\author{
$\operatorname{AUTHOR}(\mathrm{S}):$ \\ Tanimoto, Takashi; Nakano, Takato
}

\section{CITATION:}

Tanimoto, Takashi ... [et al]. Stress relaxation of wood partially non-crystallized using aqueous NaOH solutions. Carbohydrate Polymers 2012, 87(3): 2145-2148

ISSUE DATE:

2012-02

URL:

http://hdl.handle.net/2433/152368

\section{RIGHT:}

(c) 2011 Elsevier Ltd.; この論文は出版社版でありません。引用の際には 出版社版をご確認ご利用ください。; This is not the published version. Please cite only the published version. 
1

\section{Stress relaxation of wood partially non-crystallized} using aqueous $\mathrm{NaOH}$ solutions

\author{
Takashi Tanimoto ${ }^{1}$
}

Takato Nakano $2^{*}$

1: Laboratory of Biomaterials Design, Division of Forest and Biomaterials Science, Graduate School of Agriculture, Kyoto University, Kita-Shirakawa, Kyoto, 606-8502 Japan

*: corresponding author

Laboratory of Biomaterials Design, Division of Forest and Biomaterials Science, Graduate School of Agriculture, Kyoto University, Kita-Shirakawa, Kyoto, 606-8502 Japan

Tel.:+81-75-753-6234

.e-mail address; tnakano@kais.kyoto-u.ac.jp. (18)

Keywords:

Stress relaxation

Relaxation rate

X-ray diffraction

crystallinity

$\mathrm{NaOH}$ solution

\section{High lights}

$>$ We examine the effect of the crystallinity on the mechanical properties of wood. > The relaxation modulus and relaxation rate decreased with decreasing crystallinity. > The crystallinity dependence on the relaxation rate increased with increasing time. > Non-crystallized regions formed along the microfibril longitudinal axis. 
36

37

38

\section{Abstract}

Wood samples (Picea jezoensis Carr.) were treated with aqueous $\mathrm{NaOH}$ solutions (0-0.20 concentration fraction, 12 conditions), and bending tests were performed to measure stress relaxation. The relationship between mechanical properties and $\mathrm{NaOH}$ concentration is discussed. The relaxation modulus and relaxation rate were divided into three concentration ranges. Both decreased slightly for $\mathrm{NaOH}$ concentrations less than 0.10 , decreased drastically for concentrations between 0.11 and 0.14 , and decreased slightly for concentrations greater than 0.15 . The change in relaxation behavior upon $\mathrm{NaOH}$ treatment was due to an increase in molecular chain mobility in non-crystallized regions along the microfibril longitudinal axis in wood as well as lignin swelling. Furthermore, the molecular chain response in this region required time; thus, the dependence of crystallinity on the relaxation rate was apparent in the long time region. 


\section{Introduction}

Wood treated with aqueous $\mathrm{NaOH}$ shows an anisotropic dimensional change, especially along the longitudinal axis, and the contraction depends on the alkali concentration. The researcher who discussed this phenomenon in detail for the first time was Stöchmann (1971a, 1971b), who pointed out the need for microfibril contraction based on thermodynamic considerations. Contraction is induced by $\mathrm{NaOH}$ and other alkaline solutions (Nakano, 1988a, 1988b, 1989), and the degree of contraction depends on the type and concentration of the alkaline solution and increases as $\mathrm{NaOH}<\mathrm{KOH}<\mathrm{LiOH}$ at the same concentration. Nakano et al. (2000) reported an experimental temperature dependence of longitudinal contraction and showed that contraction depends on a change in the ultrastructure of the cell wall, especially the non-crystallization of microfibrils. That is, contraction is due to a non-crystallized region along the microfibril longitudinal axis and the entropy elastic force caused by the non-crystallization of cellulose microfibril helical winding in cell walls. Moreover, Nakano (2010) formulated a relationship between anisotropic dimensional changes and the crystallinity change due to alkali treatment.

The treatment of native cellulose with $\mathrm{NaOH}$ aqueous solution has been well-known as mercerization and industrially used so long time. Cellulose exhibits various polymorphism with $\mathrm{NaOH}$ treatment (Okano et al., 1984; Fink et al., 1985) and changes its crystallinity during their processes (Fengel et al., 1995; Zhou et al., 2003; Ouajai et al., 2004). Cellulose in wood also examined the effect of $\mathrm{NaOH}$ treatment with $\mathrm{NaOH}$ solution on the structure. Revol et al. (1981) and Shiraishi et al. (1984) reported that the cellulose structure in wood hardly changed due to matrix consisted of lignin and hemicellulose. Most of these studies were examined at higher concentration of $\mathrm{NaOH}$.

The mechanical behavior of alkali-treated wood also depends on the concentration of the alkaline solution. Stress-strain behavior depends on the alkali concentration and shows marked changes for $\mathrm{NaOH}$ concentrations between 0.10 and 013 (Nakano, 1989, 2000). Relaxation behavior during alkali treatment has also been reported (Nakano, 1988); however, the samples were oven-dried, and the effects of collapse were not considered. Also, the change in relative crystallinity was not discussed.

In the present study, mechanical relaxation behavior and crystallinity index were measured, and the effect of wood non-crystallization by $\mathrm{NaOH}$ treatment on mechanical relaxation behavior is discussed.

\section{Experimental}


Wood samples were prepared from Yezo spuruce (Picea jezoensis Carr.) with rectangular dimensions of $70(\mathrm{~L}) \times 7.5(\mathrm{R}) \times 2(\mathrm{~T})(\mathrm{mm})$, where $\mathrm{L}, \mathrm{R}$, and $\mathrm{T}$ are the longitudinal, radial, and tangential directions, respectively. Samples were oven-dried at $60^{\circ} \mathrm{C}$ under vacuum for 1 day. The weight and dimensions of the oven-dried samples were measured; they were then soaked in aqueous $\mathrm{NaOH}$ solutions ( $0-0.20$ concentration fraction) and stored at room temperature for 2 days, after which they were washed in distilled water for 2 weeks. The weight and dimensions of the treated samples were measured again. Three samples were treated using each $\mathrm{NaOH}$ concentration, and two unwarped samples were selected: one was subjected to stress relaxation measurements and the other was analyzed using X-ray diffraction (XRD). The sample selected for stress relaxation measurements remained in distilled water until the measurements were taken.

\section{Stress relaxation measurements}

Stress relaxation measurements were conducted in water at $25^{\circ} \mathrm{C}$ using a three-point center concentrated-load bending test with a span of $50 \mathrm{~mm}$. The displacement of $0.27 \mathrm{~mm}$ was less than one-third of the proportional displacement limit for the samples, which was applied to the LR-plane. The measurement period was about 24 hours, and the relaxation modulus was calculated as a function of time based on the measurement load.

\section{$X$-ray analysis}

Alkali-treated samples were freeze-dried under vacuum overnight after being frozen with liquid nitrogen. Freeze-dried samples were oven-dried at $60^{\circ} \mathrm{C}$ under vacuum over $\mathrm{P}_{2} \mathrm{O}_{5}$ for 1 day. The diffraction patterns were obtained for the LR-plane at room temperature by a Rigaku Ultima IV, set to $40 \mathrm{kV}$ and $40 \mathrm{~mA}$ in the scanning range of $5-35^{\circ}$. The relative crystallinity of the samples was calculated from the diffraction profile in the range of $10-28^{\circ}$. That is, X-ray diffraction profile was isolated to a crystalline part and a non-crystalline part using commercial software the PeakFit (SeaSolve Software, Inc.) for peak isolation and then each area and the whole area were calculated. The relative crystallinity was calculated as the proportion of the area of crystalline part to the whole area of the scattering area (Iwamoto et al. 2007).

\section{Results and Discussion}

Studies on the characteristic changes in mechanical behavior and dimension after alkali treatment have reported that structural changes in wood microfibrils are a key factor (Nakano, 1988, 1989, 2000, 2010). Moreover, the relative crystallinity of wood microfibrils reportedly decreases due to alkali 
treatment. Additionally, wood microfibrils do not undergo a lattice transformation from cellulose I to cellulose II; the cellulose alone changes. Thus, we start by discussing the lattice transformation of the samples.

Lattice transformation during alkali treatment is discussed based on XRD analysis. Figure 1 shows typical X-ray diffractograms, and Figure 2 illustrates the relationship between the $\mathrm{NaOH}$ concentration and the relative crystallinity. A lattice transformation from cellulose I to cellulose II during alkali treatment is well-known to not occur in wood (Revol et al. (1981), Shiraishi et al. (1984)), and Figure 1 confirms that the lattice transformation did not take place. However, the relative crystallinity was nearly constant for concentrations less than 0.10 but decreased with increasing concentration above 0.11. This dependence on alkali concentration is consistent with previous reports (Ishikura, 2010; Nakano, 2010).

The proportion of microfibrils and the fibril angle influence the mechanical properties of wood. Fujimoto et al. (2000) reported that the fibril angle increased slightly for $\mathrm{NaOH}$ concentrations between 0.10 and 015 in alkali-treated Yezo spruce samples, and Nakano (2010) stated that the fibril angle changed little during alkali treatment. In the present study, the change in the fibril angle appears to be slight. Based on the study of Reiterer et al. (1999) on the relationship between Young's modulus and fibril angle, our result was probably due to a decrease in crystalline cellulose, which has a high Young's modulus. Thus, the change in fibril angle is neglected in the following discussion.

Figure 3 shows relaxation curves for each $\mathrm{NaOH}$ concentration. Plots of $\mathrm{E}(\mathrm{t}) \mathrm{vs}$. $\ln (\mathrm{t})$ did not show a clear difference in relaxation behavior, but the relaxation modulus displayed characteristic concentration dependence.

Previous studies have demonstrated that the concentration dependence of the relaxation modulus was due to an internal structural change during alkali treatment. Figure 4 shows the dependences of the relaxation modulus at $\ln (\mathrm{t})=0$ and the dimensional change along the longitudinal axis on $\mathrm{NaOH}$ concentration and the degree of crystallinity. Both show similar concentration and crystallinity dependence, indicating that both changes were due to the same factor, i.e., the change in relative crystallinity.

The concentration dependence of the relaxation modulus in Figure 4 was divided into three ranges. The relaxation modulus decreased slightly for $\mathrm{NaOH}$ concentrations less than 0.10 , decreased considerably for concentrations between 0.11 and 0.14 , and decreased slightly for concentrations greater than 0.15 . This result is consistent with a previous report (Nakano et al., 2000). Nakano et al. (2000) showed that the longitudinal contraction in wood during alkali treatment was due to a non-crystallized region along the microfibril longitudinal axis, and that contraction was due to the entropy elastic force because of an increase in the heating temperature. Additionally, Nakano (2010) reported that anisotropic swelling during alkali treatment can be explained by the 
aforementioned mechanism. Similar relationships between the relaxation modulus and the dimensional change along the longitudinal axis on the $\mathrm{NaOH}$ concentration and the degree of crystallinity are consistent with the above discussion.

Considering the change in relative crystallinity, a decrease in the relaxation modulus for $\mathrm{NaOH}$ concentrations less than 0.10 (Fig. 4) was not due to the change in relative crystallinity. It probably occurred because of an increase in the geometrical moment of inertia during alkali treatment, which increased linearly for $\mathrm{NaOH}$ concentrations less than 0.10 and was nearly constant for concentrations greater than 0.11 .

The concentration dependence of the relaxation modulus in Figure 3 suggests that non-crystallization due to alkali treatment affected the relaxation modulus during measurements. This makes comparison among the relaxation spectra difficult because the relaxation modulus changed drastically during alkali treatment, and comparing the change in relaxation behavior using an absolute value is difficult. Thus, relaxation behavior was evaluated based on the relaxation rate, $d \ln E(t) / d \ln t$. This value represents the relaxation level at each time, making the change in relaxation behavior clear.

Figure 5 shows $d \ln E(t) / d \ln t$ vs. $\ln (\mathrm{t})$. The relaxation rate was divided into three concentration ranges: non-alkali treated (0), 0.03-0.12, and greater than 0.13 . The concentration ranges correspond to the three ranges in Figure 4. That is, the relaxation behavior changed due to non-crystallization during alkali treatment. Figure 5 shows that the absolute relaxation rate increased in the long time region for $\mathrm{NaOH}$ concentrations less than 0.12 , and that the rate increased in the short and long time regions for concentrations greater than 0.13 .

The relationship between the relaxation rate and relative crystallinity is shown in Figure 6 . The relaxation rate changed markedly for relative crystallinities between 0.30 and 0.33. Although the relaxation modulus changed considerably, the relaxation rate changed slightly in the short time region but significantly in the long time region. The relaxation rate at $\ln (\mathrm{t})=11$ changed considerably for relative crystallinities between 0.30 and 0.33 . Additionally, the long-term relaxation rate recovered for relative crystallinities of less than 0.30 . For relative crystallinities greater than 0.33 or for $\mathrm{NaOH}$ concentrations less than 0.12 , the relaxation rate changed little, regardless of time.

The mechanism of the change in relaxation behavior with decreasing relative crystallinity is discussed. Two mechanisms must be considered: one is a crystallinity dependence on the relaxation modulus (Fig. 4), and the other is a crystallinity dependence on the relaxation rate (Fig. 6). The former is not time-dependent, but the latter is.

The former increases the region reacting to deformation promptly, namely the non-crystallized region. That is, the decrease in the relaxation modulus at $\ln (\mathrm{t})=0$ with increasing $\mathrm{NaOH}$ concentration was due to a decrease in the fraction of the crystallized region (Fig. 4), which was 
rigid, and an increase in the fraction of the non-crystallized region, which was flexible. Another factor is a decrease in the contribution of lignin, which binds microfibrils. The interaction between microfibrils and lignin is believed to have been weakened by swelling during alkali treatment. Alkali solution causes dissolution of hemicellulose, but dissolution was nearly complete at a $\mathrm{NaOH}$ concentration of 0.05 and did not increase for concentrations greater than 0.05 . Thus, dissolution scarcely affected the mechanical properties.

The relaxation rate depended on the response of molecular chain rearrangement. Figure 6 shows that the crystallinity dependence on the relaxation rate increased with increasing $\ln (\mathrm{t})$, suggesting that molecular chain rearrangement required time, and that the change in the relaxation rate related to the restricted molecular chain motion. Furthermore, the relaxation rate did not change for relative crystallinities greater than 0.33 but did change for relative crystallinities less than 0.33, suggesting that the molecular chain response required a large non-crystallization region. Thus, non-crystallized regions formed along the microfibril longitudinal axis. The motion of non-crystallized regions around the microfibrils appears to be restricted by crystalline regions parallel to the longitudinal axis.

Nakano et al. (2000) proposed that a reduction in the end-to-end distance of the chain segments in the amorphous regions was due to longitudinal contraction of microfibrils caused by alkali solution penetrating the defects in the microfibrils longitudinal direction. For the experimental method used in this study (three-point bending tests), contractive and tensional stress occurred. The S2-layer, which is about 0.80 fraction occupation of the cell wall, so that it dominates the mechanical properties of wood. The result shown in Figure 4 suggests that the alkali solution penetrated the defects in the microfibril longitudinal direction, and that a non-crystallized region was formed along the longitudinal axis for softwood, in which the fibril angle of the S2-layer is small. Furthermore, molecular chain rearrangement in this region requires time; thus, the crystallinity dependence on the longer time relaxation rate (Fig. 6) was evident.

\section{Conclusion}

The effect of the crystallinity change induced by $\mathrm{NaOH}$ treatment on the mechanical relaxation behavior of wood was investigated. The relaxation modulus and relaxation rate were divided into three concentration ranges. Both decreased slightly for $\mathrm{NaOH}$ concentrations less than 0.10 , decreased markedly for concentrations between 0.11 and 0.14 , and decreased slightly for concentrations greater than 0.15 . This was due to an increase in the molecular chain mobility in non-crystallized regions along the microfibril longitudinal axis in wood and lignin swelling due to the 
$224 \mathrm{NaOH}$ treatment. Furthermore, molecular chain rearrangement in this region requires time; thus, 225 the crystallinity dependence on the longer time relaxation rate was apparent.

226 


\section{References}

Fengel D., Jakob H. , and Storobel C.(1995). Influence of the Alkali Concentration on the Formation of Cellulose. Horzforschung 49, 505-511

Fink H. P. and Philipp B.(1985). Models of Cellulose Physical Structure from the Viewpoint of the Cellulose I $\rightarrow$ II transition. J. Applied Polymer Science, 30, 3779-3790.

Fujimoto T., Nakano T.(2000). The effect of mercerization on wood structural feature. Mokuzai Gakkaishi, 46(3), 238-241

Ishikura Y., Abe K., and Yano H. (2010). Bending Properties and cell wall structure of alkari-treated wood. cellulose, 17, 47-55

Iwamoto W., Nakagaito A. N., and Yano H. (2007). Nano-fibrillation of pulp fibers for the processing of transparent nanocomposites. Appl. Phys. A89, 461-466

Nakano T.(1988a). Plasticization of wood by alkali treatment. Journal of the Society of Rheology Japan, 16, 104-110

Nakano T.(1988b). Plasticization of wood by alkali treatment. Effects of kind of alkali and concentration of alkali ne aqueous solution on stress relaxation. Mokuzai Gakkaishi, 16, 104-110

Nakano T.(1989). Plasticization of wood by alkali treatment. Relationship between plasticization and ultra-structure. Mokuzai Gakkaishi, 35, 431-437

Nakano T., Sugiyama J., and Norimoto M.(2000). Contractive Force and Transformation of Microfibril with Aqueous Sodium Hydroxide Solution for Wood. Horzforschung, 54, 315-320

Nakano T. (2010). Mechanism of microfibril contraction and anisotropic dimensional changes for cells in wood treated with aqueous $\mathrm{NaOH}$ solution. Cellulose, 17, 711-719.

Okano T. and Sarko A.(1984). Mercerization of Cellulose. I. X-ray Diffraction Evidence for Intermediate Structures. J. Applied Polymer Science, 29, 4175-4182.

Ouajai S., Hodzic A., Shanks R.A.(2004). Morphological and Grafting Modification of Natural Cellulose Fibers. J. Applied Polymer Science, 94, 2456-2465.

Reiterer A., Lichtenegger H., Tschegg S., Fratzl P.(1999). Experimental evidence for a mechanical function of the cellulose microfibril angle in wood cell walls. Philos. Mag, A79 (9),2173-2184

Revol J. F. and Goring D. A. I.(1981). On the Mechanism of the Mercerization of Cellulose in Wood. J. Applied Polymer Science, 26, 1275-1282.

Shiraishi N., Moriwaki M., Lonikar S. V. and Yokota T.(1984). Lattice Conversion of Cellulose in Wood. J. Wood Chemistry and Technology, 4(2), 219-238.

Stöckmann V. E. (1971a). Effect of Pulping on Cellulose Stucture Part1. A Hypothesis of Transformation of Fibrils. Tappi, 54(12), 2033-2037

Stöckmann V. E. (1971b). Effect of Pulping on Cellulose Stucture Part2. Fibrils Contract Longitudinally. Tappi , 54(12), 2038-2045 
262

263

264

265

266

Zhou L. M., Yeung K. W., Yuen C. W. M., Zhou X.(2004). Characterization of Ramie Yarn Treated with Sodium Hydroxide and Crosslinked by 1,2,3,4-Butanetetracarboxylic Acid. J. Applied Polymer Science, 92, 1857-1864. 


\section{$267 \quad$ Figure captions}

Figure 1. Typical X-ray diffractograms of untreated and treated wood using aqueous $\mathrm{NaOH}$ solutions.

Figure 2. Relationship between crystallinity and $\mathrm{NaOH}$ concentration.

Figure 3. Stress relaxation curves of samples treated with various $\mathrm{NaOH}$ solutions. Note: numbers indicate $\mathrm{NaOH}$ concentration fractions.

Figure 4. Dependence of the relaxation modulus at $\ln (\mathrm{t} / \mathrm{s})=0$ and the dimensional change along the longitudinal direction on $\mathrm{NaOH}$ concentration and relative crystallinity.

Figure 5. Relaxation rate, $d \ln E(t) / d \ln [t]$, of samples treated with various $\mathrm{NaOH}$ concentrations.

Figure 6. Dependence of the relaxation rate at $\ln (\mathrm{t} / \mathrm{s})=3,8$, and 11 on relative crystallinity. 
283

284

285

286

287

288

289

290

291

292

293

294

295

296

297

298

299

300

301

302

303

304

305

306

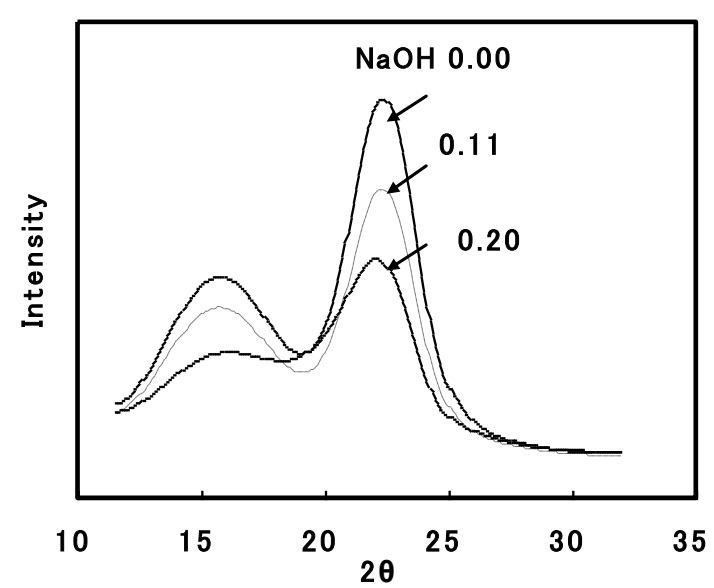

Figure 1. Typical X-ray diffractograms of untreated and treated wood using aqueous $\mathrm{NaOH}$ solutions. 


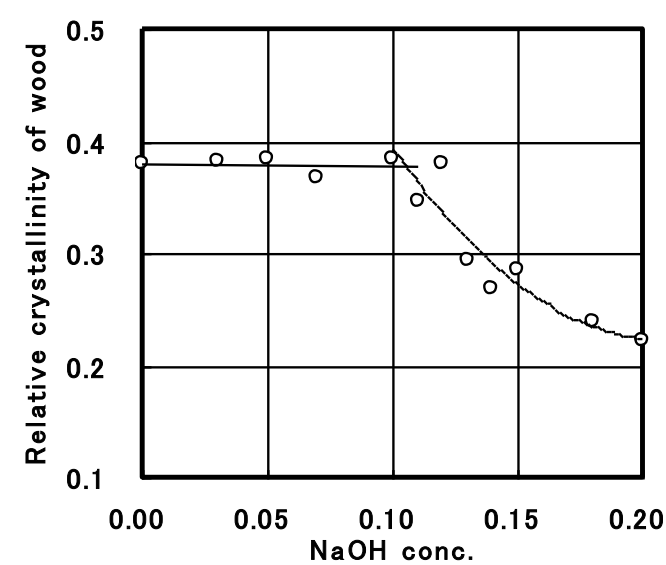

325

326

327

Figure 2. Relationship between crystallinity and $\mathrm{NaOH}$ concentration.

328

329 


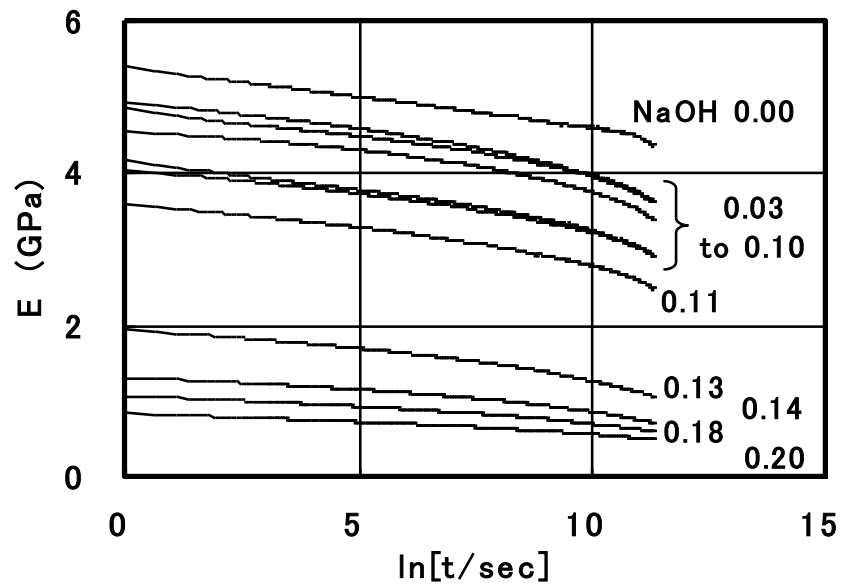

348

349 Figure 3. Stress relaxation curves of samples treated with various $\mathrm{NaOH}$ solutions. Note: numbers 350 indicate $\mathrm{NaOH}$ concentration fractions.

351

352 
6
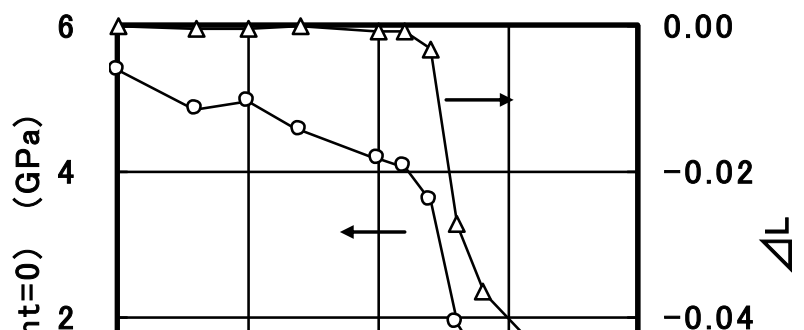

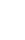
67 69 1

Figure 4. Dependence of the relaxation modulus at $\ln (\mathrm{t} / \mathrm{s})=0$ and the dimensional change along the 379 longitudinal direction on $\mathrm{NaOH}$ concentration and relative crystallinity.

380

381 
382

383

384

385

386

387

388

389

390

391

392

393

394

395

396

397

398

399

400

401

402

403

404

405

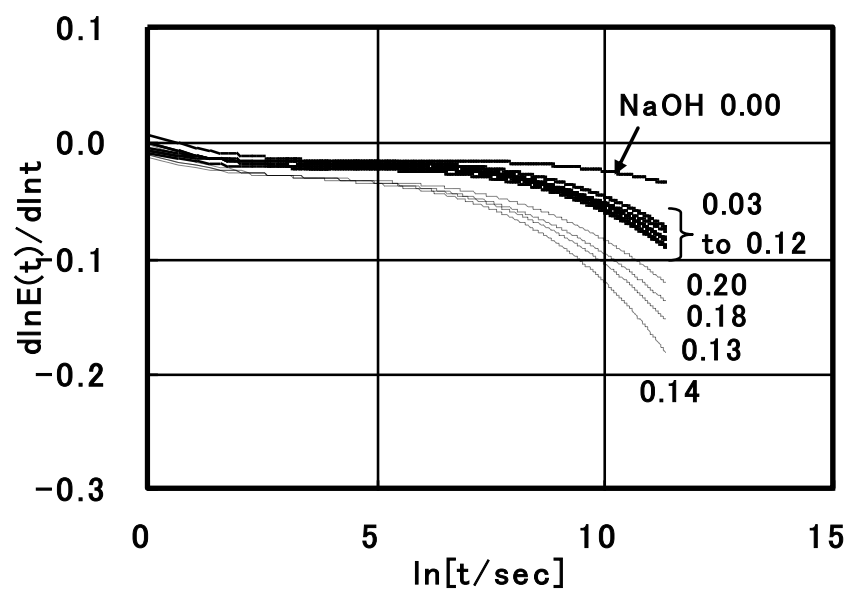

Figure 5. Relaxation rate, $d \ln E(t) / d \ln [t]$, of samples treated with various $\mathrm{NaOH}$ concentrations. 
406

407

408

409

410

411

412

413

414

415

416

417

418

419

420

421

422

423

424

425

426

427

428

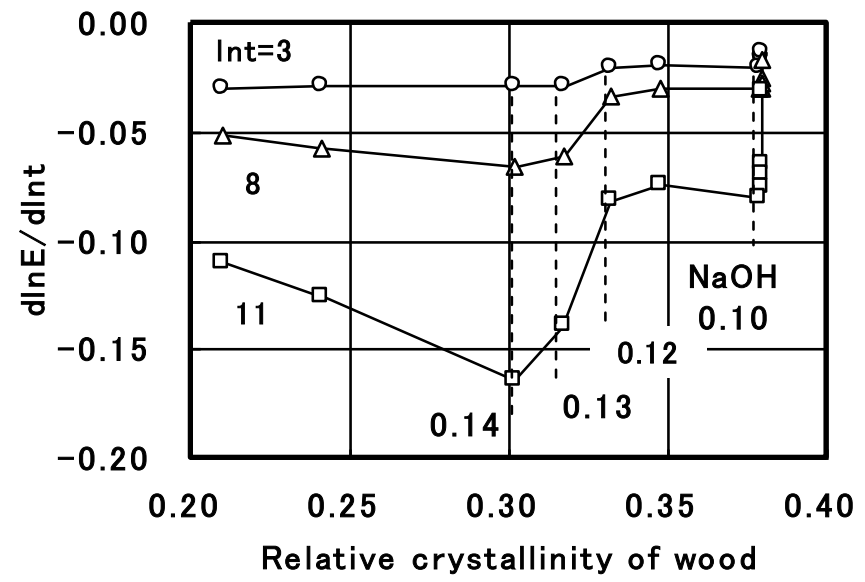

Figure 6. Dependence of the relaxation rate at $\ln (\mathrm{t} / \mathrm{s})=3,8$, and 11 on relative crystallinity. 